\title{
SUMMARY OF THESIS
}

\author{
TOLEDO, Max Jean de Ornelas - Comportamento biológico em camundongos de clones de Trypanosoma cruzi pertencentes a diferentes \\ genótipos. Belo Horizonte, 2001. (Tese de Doutorado - Instituto de Ciências Biológicas da Universidade Federal de Minas Gerais).
}

\section{BIOLOGICAL BEHAVIOR IN MICE OF DIFFERENT Trypanosoma cruzi CLONAL GENOTYPES}

Twenty Trypanosoma cruzi laboratory-cloned stocks attributed to the 19 and 20 clonal genotypes included in T. cruzi I (Satellite Meeting, Rio de Janeiro, 1999) and to the 39 and 32 clonal genotypes included in T. cruzi II were comparatively studied in BALB/c mice during the acute and chronic phases of the infection to test the working hypothesis that $T$. cruzi clonal evolution has a major impact on its biological properties. Twenty two parameters were assayed: (i) infectivity, pre patent period, patent period, maximum of parasitemia, day of maximum of parasitemia and area under the curve of parasitemia, during the acute phase of the infection; (ii) mortality, \% of positive hemoculture, tissue parasitism, inflammatory process, $\%$ of positive ELISA, $\%$ of positive anti-live trypomastigotes antibody (ALTA), susceptibility to benznidazole (BZ) and susceptibility to itraconazole (ITZ), during both acute and chronic phases of the infection.

The stocks pertaining to the clonal genotype 19, phylogenetically very close to the clonal genotype 20 and distinct from it by only one enzyme locus (6-phosphogluconate dehydrogenase - 6Pgdh) out of 22 loci analyzed, were similar in the majority (16 out of 22) of the biological parameters studied. They were significantly different in six important biological variables related to parasitemia pattern and BZ susceptibility. The clonal genotype 19 was considered of intermediate resistance to BZ and totally resistant to ITZ.

Mice infected with stocks attributed to the clonal genotype 20 displayed the highest values for 8 parameters and the lowest values in 2 parameters out of 22 analyzed from all genotypes. Stocks attributed to the clonal genotype 20 displayed values significantly higher for infectivity, area under the curve of parasitemia, maximum of parasitemia, tissue parasitism and inflammatory process in the acute phase. Thus, they were considered as more virulent and pathogenic during the acute phase of the infection and totally resistant to both chemotherapeutic agents in both phases of the infection.

Stocks attributed to the clonal genotype 39 exhibited the most contrasting parasitemia levels. Mice infected with this genotype were the only ones in which tissular parasitism was not detected in the acute phase and in which tissular parasitism was detected in the chronic phase of the infection. Only mice infected with stocks of this clonal genotype showed no association between the levels of tissular parasitism and inflammatory process. This genotype was considered the most susceptible to host immune response and of intermediate resistance to the drugs under study, since it showed sensitive, partially resistant and totally resistant stocks.

Mice infected with stocks attributed to the clonal genotype 32 exhibited the lowest values for in 8 out of 18 parameters related to virulence, pathogenicity and humoral immune response from all genotypes. Thus, this genotype was considered the less virulent among all the genotypes studied and induces low antibodies production. This genotype was considered sensitive to both chemotherapeutic agents.

Statistical analysis showed that the results are overall consistent with the working hypothesis: biological differences are proportional to the evolutionary divergence among the genotypes. Thus, closely related genotypes (19 vs 20 and 32 vs 39) show fewer differences than distantly related genotypes (19 or 20 vs 32 or 39 ). The working hypothesis is further supported by the result of the nonparametric Mantel test, which showed a highly significant correlation $\left(r=0.4233, \mathrm{p}=10^{-4}\right)$ between biological differences and genetic distances among all pairs of stocks. However, although this correlation is highly significant, some stocks show non-typical biological behaviors, and tend to be similar to stocks from other genetic groups, as shown by cluster analysis of the 22 genetic markers and from the 17 biological parameters. Interestingly, it was observed that genotypes 19 and 20 were different in six important biological variables out of 22 although they are genetically very close.

Our results confirm the statistical link between genetic divergence and biological behavior and strongly suggest that the 2 major subdivisions T. cruzi I and II are too broad for biological studies and that it is relevant to take into account also the lesser genetic groups, in all applied studies dealing with diagnosis, drug and vaccine design, epidemiological surveys and clinical diversity of Chagas' disease.
Max Jean de Ornelas Toledo mjotoledo@uem.br 